\title{
Model Predictive Load-Frequency Control Taking into Account Imbalance Uncertainty
}

\author{
Anne Mai Ersdal ${ }^{\mathrm{a}, *}$, Lars Imsland ${ }^{\mathrm{a}}$, Kjetil Uhlen ${ }^{\mathrm{b}}$, Davide Fabozzic ${ }^{\mathrm{c}}$, Nina F. \\ Thornhill ${ }^{\mathrm{c}}$ \\ ${ }^{a}$ Department of Engineering Cybernetics \\ Norwegian University of Science and Technology, O.S. Bragstadsplass 2D, \\ 7034 Trondheim, Norway \\ (e-mail: \{anne.mai.ersdal,lars.imsland\}@itk.ntnu.no). \\ ${ }^{b}$ Department of Electric Power Engineering \\ Norwegian University of Science and Technology, Trondheim, Norway \\ (e-mail: kjetil.uhlen@ntnu.no). \\ ${ }^{c}$ Department of Chemical Engineering \\ Imperial College London, London, United Kingdom \\ (e-mail: davide.fabozzi@gmail.com,n.thornhill@imperial.ac.uk).
}

\begin{abstract}
Nonlinear model predictive control (NMPC) is investigated for load frequency control (LFC) of an interconnected power system which is exposed to increasing wind power penetration. The robustified NMPC (RNMPC) proposed here uses knowledge of the estimated worst-case deviation in wind-power production to make the NMPC more robust. The NMPC is based on a simplified system model that is updated using state- and parameter estimation by Kalman filters, and takes into account limitations on among others tie-line power flow. Tests on a proxy of the Nordic power system, shows that the RNMPC is able to fulfill system constraints under worst-case deviations in wind-power production in cases where the nominal NMPC is not.
\end{abstract}

Keywords: Model Predictive Control, Load Frequency Control, Robust Control, Intermittent Energy Resources, Power System Control

\footnotetext{
*Corresponding author.

Email address: anne.mai.ersdal@itk.ntnu.no (Anne Mai Ersdal)
} 


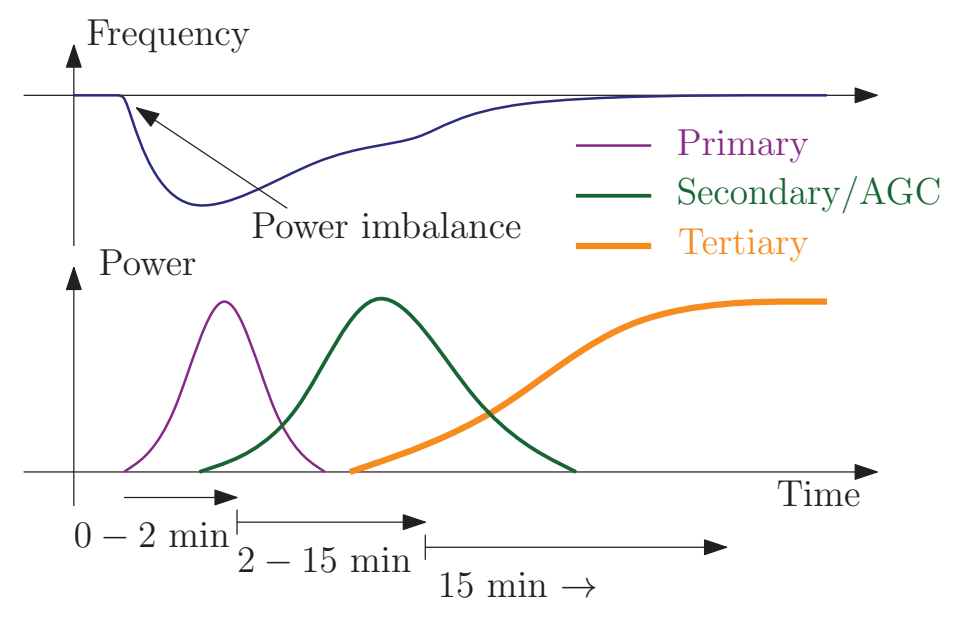

Figure 1: Activation of primary, secondary (AGC) and tertiary control after a power imbalance.

\section{Introduction}

Power systems around the world have been through great development during the last two decades. First with the liberalization of the power markets in the 1990's, and second with the increasing amount of renewable energy resources, distributed generation, and increasing energy need seen around the world. These are all elements which cause challenges for the operation of power systems, and especially with regards to load frequency control (LFC).

LFC is a term applied to describe the continuous operation of keeping the frequency of a power system stable. The frequency of a power system is connected to the balancing of produced and consumed power in the way that if there is a surplus of produced power the frequency will rise, and if there is a lack of produced power the frequency will fall. It is very important that this power balance is maintained, if not the generators could lose synchronism, and the power system would collapse. Traditionally, LFC has a hierarchical structure with primary, secondary, and tertiary control ${ }^{1}$, see Figure 1. Primary control is continuous, automatic control placed locally at the generators. It is often based on proportional control, and it instantaneously covers the power imbalance between produced and consumed power. It does

\footnotetext{
${ }^{1}$ Also known as frequency containment reserves (FCR), frequency restoration reserves (FRR), and replacement reserves (RR).
} 


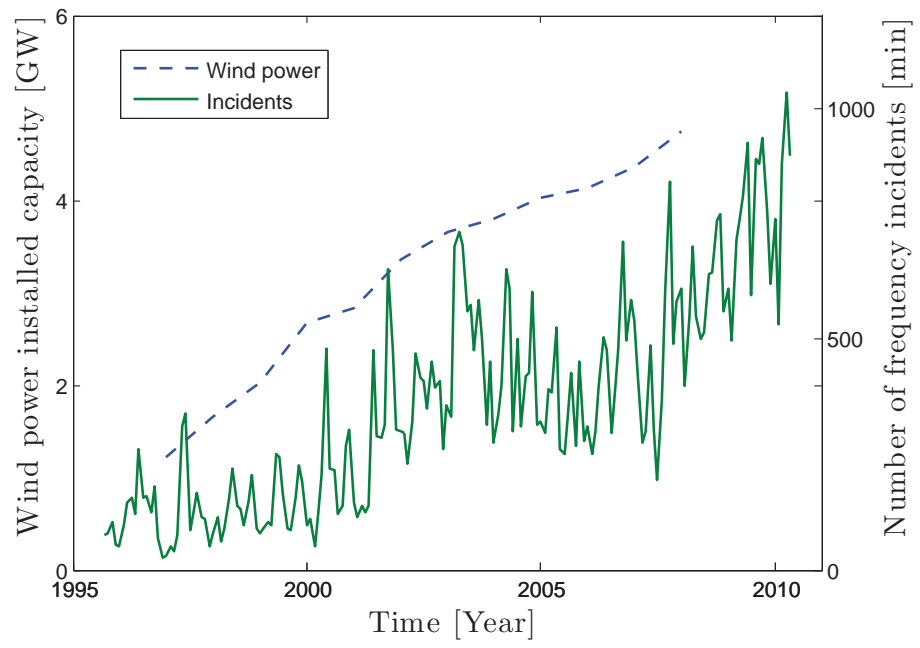

Figure 2: Number of frequency incidents per month (Whitley and Gjerde, 2011) and installed wind power capacity (Nordel, 2008) in the Nordic system.

not, however, ensure that the frequency is restored to its set point. For this, secondary control is needed. Secondary control is a slower, centralized, and automatic controller which releases primary control. It is often referred to as automatic generator control (AGC), and this term will be applied in the following. Tertiary control is an even slower, centralized controller, which again releases the AGC. This is manually operated by the transmission system operator (TSO). In the Nordic network, consisting of Norway, Sweden, Finland, and the eastern parts of Denmark, hydro generators are the main provider for primary control, while other generating units such as thermal and nuclear power generators as well as some controllable loads participate in tertiary control (Statnett, 2012). AGC was first implemented here in 2012/2013, and it is assumed that hydro generators will be the main provider for this as well.

In the Nordic Network, the TSOs aim at keeping the frequency between 49.9 and $50.1 \mathrm{~Hz}$. This is something that has proven to be increasingly difficult, and as seen from Figure 2, the number of frequency incidents (minutes spent outside 49.9 and $50.1 \mathrm{~Hz}$ ) has increased concurrently with installed wind power capacity over the last decade. It is confirmed by Statnett, the Norwegian TSO, that the increasing amount of intermittent energy resources is part of the reason for the decreasing control performance, along with a heavier loaded network and an increasing amount of bottlenecks, which at times excludes some of the resources from participating in LFC (Statnett, 
2012).

There have been many suggestions to how LFC can be improved to better cope with these challenges. In Short et al. (2007) and Fabozzi et al. (2013) loads are included in LFC, while Suvire et al. (2012) concentrate on effective energy storage, and Chang-Chien et al. (2011) suggests how wind generators can participate in LFC. Others concentrate on new control methods for LFC, such as including primary control in local decentralized generators (Marinovici et al., 2013), or improving LFC through fuzzy logic (Yousef et al., 2014), sliding mode control (Vrdoljak et al., 2010), internal model control (Saxena and Hote, 2013), and various PID tuning methods (Tan, 2010). Many have also investigated model predictive control (MPC) as a way of improving LFC, e.g. through building climate control (Halvgaard et al., 2012), or control of power flows in high voltage direct current (HVDC) lines (McNamara et al., 2013). In Otomega et al. (2007); Carneiro and Ferrarini (2010) MPC is used as a special protection scheme to prevent severe line-overloads.

Some have also investigated MPC for AGC, for example Venkat et al. (2008); Shiroei et al. (2013); Mohamed et al. (2012). However, none of these consider model-plant mismatch. Others discuss robust MPC (Shiroei et al., 2013), but this is mainly against system parameter uncertainties, and the fulfillment of system constraints is not considered. Other control methods have also been suggested for robust LFC, such as $H_{\infty}$ control (Singh et al., 2013; Bevrani et al., 2011), fuzzy logic (Çam and Kocaarslan, 2005), and robust PD tuning methods (Khodabakhshian and Edrisi, 2008).

Rather generally, robust MPC is formulated in a min-max framework, where the optimization seek to find minimizing inputs for the disturbances that maximize the objective function. See for example Rawlings and Mayne (2009), Mayne et al. (2000) (nonlinear systems), Löfberg (2003) for linear systems, and various approaches to ease the computational load, e.g. based on precomputation of invariant sets (e.g. tube-based MPC (Langson et al., 2004)). Despite recent progress, min-max MPC still gives prohibitive complexity as problem dimensions grow. In this paper, we choose a simple approximation as the maximizing disturbances that gives reasonable computational performance, but will not give recursive feasibility and guaranteed stability in general. This paper shows that this approach will still be able to handle many, or most, disturbances in the proposed setup.

In Ersdal et al. (2015) a nonlinear MPC (NMPC) based on a simplified system model was designed for AGC of the Nordic power system and tested against conventional PID-controllers. Through both descriptive examples 
and Monte Carlo simulations, it was shown that an NMPC can be beneficial in AGC, both with regards to control performance and reserve usage. It was also shown how the NMPC can take system constraints into account, such as limitations on tie-line power transfers. This is something which is not easily done with a PID controller. Ersdal et al. (2014) presented an NMPC for AGC which is robustified against variations in produced wind power. This robustified NMPC (RNMPC) was tested without any model-plant mismatch, and it showed how the RNMPC was able to plan production so that it could handle a worst-case wind-power production scenario without breaking any system constraints.

This paper is an extension of the work presented in Ersdal et al. (2014, 2015).The RNMPC from Ersdal et al. (2014) is implemented and tested with the proxy system and prediction model from Ersdal et al. (2015), hence there is a more realistic test of the controller on a large, realistic simulator. Ersdal et al. (2015) presented a complete solution to the LFC problem by including generator participation-factors in the optimization problem as well as state estimation for full state feedback. This paper will be an extension of this by including knowledge of worst-case predictions of future wind power production to robustify the NMPC. If the RNMPC can plan so that there always is enough transfer capacity on important tie-lines to handle some worst-case scenario, all generators are able to participate in LFC at all times, which will improve the system frequency.

In this work a centralized MPC (CMPC) is implemented, i.e. it is based on a model of the full system and it controls all the controllable system inputs. There are several examples where distributed MPC (DMPC) has been applied for LFC, such as Venkat et al. (2008); Mohamed et al. (2011). In DMPC separate MPCs, based on single-area models, controls the inputs to each area, and the main benefits are less demands for communication and smaller optimization problems for each MPC, hence shorter optimization time. The main drawback of DMPC is that it may result in poor systemwide control performance if the subsystems interact significantly (Venkat et al., 2008). For large power systems, such as the Central European system or the Eastern Interconnection in North America, the benefits of DMPC are very relevant. With a considerable smaller network, such as the Nordic network, on the other hand, there is not that much to gain by applying DMPC compared to CMPC, especially when considering that the CMPC presented here is based on a simplified model with a rather small amount of optimization variables. In addition to this, using a DMPC would complicate the tie-line constraint 
handling, which is an important feature of the presented controller.

The remainder of this paper is organized as follows. In Section 2 the proxy system used to represent the Nordic power system and the prediction model used in the NMPC are presented. The nominal NMPC (NNMPC) is then discussed in Section 3, before the details of the RNMPC are given in Section 4. In Section 5 the case study is presented along with the results from the tests on the proxy system, which then are discussed in Section 6 . Final conclusions on the presented work are given in Section 7.

\section{Modeling}

The proxy system and the prediction model used in this work are the same as the ones used in Ersdal et al. (2015). This section is therefore a condensed version of Section II of Ersdal et al. (2015). All variables, in both the proxy system and the prediction model, are given in per unit [p.u.], see Appendix A in Machowski et al. (2008) for details.

The transmission grid is a critical infrastructure which cannot be used as a test-bed. For research in grid transmission control, it is therefore always necessary to use a simulation as a proxy for the physical system. The power system model used here is a model of the Nordic power system developed by SINTEF Energy Research (Norheim et al., 2005). It includes 15 hydro generators, 5 non-hydro generators, 21 composite loads, and 36 nodes. The placement of the generators can be seen in Figure 3, and they are chosen so that the model reflects the real production and most interesting bottlenecks in the Nordic power system (Norheim et al., 2005).

\subsection{SINTEF Proxy System}

In general a power system can be modeled by a differential algebraic equation

$$
\begin{aligned}
\dot{\mathbf{x}} & =f(\mathbf{x}, \mathbf{z}, \mathbf{u}, \mathbf{w}) \\
0 & =g(\mathbf{x}, \mathbf{z}, \mathbf{u}, \mathbf{w})
\end{aligned}
$$

where $\mathbf{x}$ are the dynamic system states, $\mathbf{z}$ the algebraic system states, $\mathbf{u}$ the controllable input and $\mathbf{w}$ the system disturbance.

The dynamics of the model consists of the electromechanical dynamics of the rotor as well as the dynamics of the turbines and their governing systems (primary control). Since it is mainly the hydro turbines which provide primary control in the Nordic network, and most likely will provide the majority 


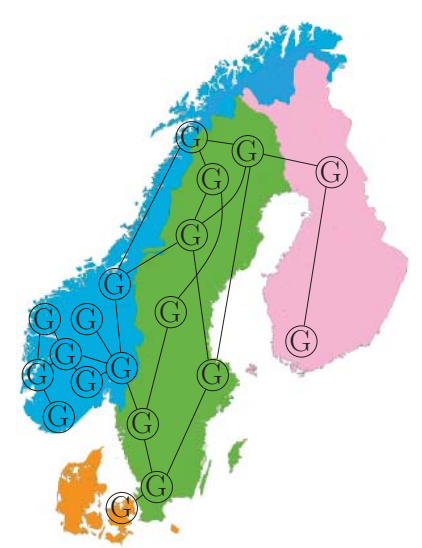

Figure 3: An overview of the generators in the SINTEF model (Norheim et al., 2005).

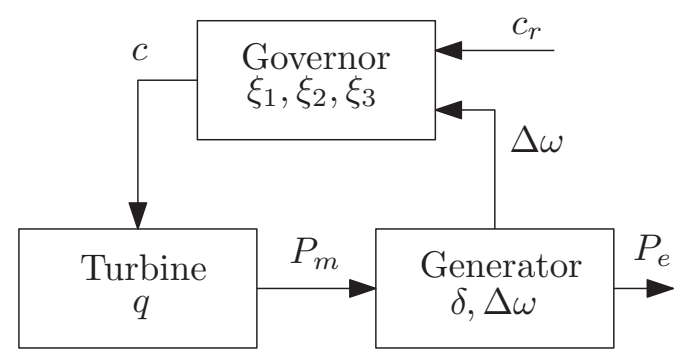

Figure 4: Overview of states, inputs and outputs of the dynamic system equation.

of AGC as well, only the hydro turbine and governor dynamics are included. Note that the rotor dynamics of the synchronous generator are modeled for all generators of the network.

Figure 4 shows a simplified diagram of the states, inputs and outputs of the dynamic system equation. Generators are modeled by what is usually denoted the swing equation, while the turbine and governor are modeled as the nonlinear model of Machowski et al. (2008), usually denoted HYGOV. The states of the swing equation are the rotor angle and angular velocity, $\delta$ and $\Delta \omega$, the state of the turbine is the penstock flowrate $q$ and the governor has the internal states $\xi_{1}, \xi_{2}, \xi_{3}$, representing the valve opening of the pilot servomotor and main servomotor as well as the integral of the controller part of the governor. The valve opening is $c=\xi_{2}$, and $c_{r}$ is the valve opening set point provided by the TSO, while $P_{m}$ and $P_{e}$ are the mechanical and electrical power outputs, respectively. It is the valve opening set-point $c_{r}$ 


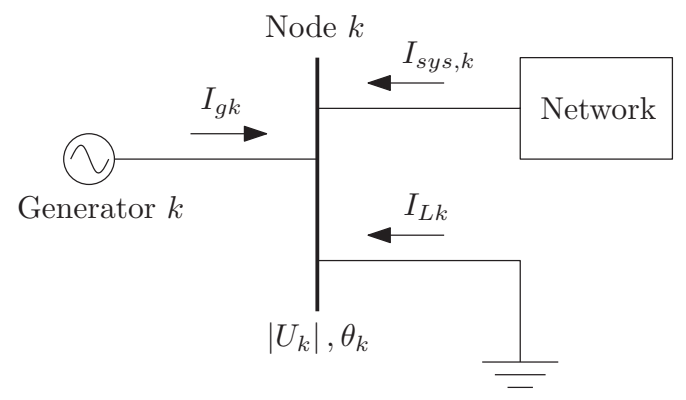

Figure 5: Internal node representation.

which will be controlled by the MPC.

The algebraic system equations describe the current flow in the network, and it is found using the internal node representation, where Kirchoff's current law is applied at each node. Figure 5 shows the currents flowing into each node, where $I_{g}$ is the current delivered from the generator, $I_{L}$ the current from the load and $I_{\text {sys }}$ the current from all the other nodes of the network. Based on the reactive power balance in the network, there is also the resulting nodal voltage $U=|U| e^{j \theta}$. When applying Kirchoff's current law in all nodes, the result is one complex equation in polar form with two unknown: $|U|$ and $\theta$, the magnitude and angle of the nodal voltages.

Given a large power system consisting of $n$ nodes and $m$ generators, where $m_{h}$ of these are hydro turbines, the total system can be written as (1) with

$$
\begin{aligned}
& \mathbf{x}=\left[\begin{array}{llllll}
\delta_{k} & \Delta \omega_{k} & q_{i} & \xi_{1 i} & \xi_{i 2} & \xi_{i 3}
\end{array}\right]^{T} \\
& \mathbf{z}=\left[\begin{array}{ll}
|U|_{s} & \theta_{s}
\end{array}\right]^{T} \\
& \mathbf{u}=c_{r i} \\
& \mathbf{w}=\left[\begin{array}{ll}
\left|I_{L s}\right| & P_{m p}
\end{array}\right]^{T}
\end{aligned}
$$

where $k=1, \cdots, m, i=1, \cdots, m_{h}, s=1, \cdots, n$, and $p=m_{h}+1, \cdots, m$. In total $2 m+4 m_{h}$ dynamic states, $2 n$ algebraic states, $m_{h}$ controllable inputs, and $n+m-m_{h}$ disturbances.

\subsection{Prediction Model for Model Predictive Control}

The prediction model (PM) is the model used by the MPC to predict how the system will behave in the future, based on the inputs to-be-optimized and a disturbance model. In real-life situations, these models are always 
simplifications and approximations to the actual processes which are being controlled. In this work, the MPC is based on a reduced and simplified version of the system presented in the previous section. This is done to ease the computational load of the MPC, but it also incurs a natural model-plant mismatch in the testing of the controller. To find the PM, the larger model of the previous section is first divided into $N$ areas connected to each other by tie lines. All states, inputs and disturbances are given relative an initial steady state where both active and reactive power supply and consumption are balanced.

The dynamics affecting the frequency response of a power system are relatively slow, and neglecting the fast dynamics reduces the complexity of the model (Bevrani, 2009). The nodal voltages and the electromechanical dynamics of the swing equation are considered to be fast dynamics, and can therefore be neglected. The dynamics of area $i$, including the generators, can in this case be represented by one single differential equation (Bevrani, 2009)

$$
\Delta \dot{\bar{f}}^{i}=\frac{1}{2 \bar{H}^{i}}\left(\Delta \bar{P}_{m}^{i}-\Delta \bar{P}_{L}^{i}-\Delta \bar{P}_{t i e}^{i}\right)
$$

where $\Delta \bar{f}$ is the deviation from the nominal frequency $f_{s}, \Delta \bar{P}_{m}$ the total change in mechanical power from primary control and AGC combined, $\Delta \bar{P}_{L}$ the total change in load power, $\Delta \bar{P}_{\text {tie }}$ the change in total power flow from the area on all its tie lines, and $\bar{H}$ the inertia of the rotating masses of the area. The change in total tie-line power flow from area $i$ is (Bevrani, 2009)

$$
\Delta \dot{\bar{P}}_{\text {tie }}^{i}=2 \pi\left(\Delta \bar{f}^{i} \sum_{j=1, j \neq i}^{N} \bar{T}_{i j}-\sum_{j=1, j \neq i}^{N} \bar{T}_{i j} \Delta \bar{f}^{j}\right)
$$

where $\Delta \bar{f}^{i / j}$ is the local frequency in area $i / j$, and $\bar{T}_{i j}$ the synchronizing torque coefficient between area $i$ and $j$. The frequency deviation of the entire system is defined as

$$
\Delta \bar{f}=\frac{\sum_{i=0}^{N} \bar{H}^{i} \Delta \bar{f}^{i}}{\sum_{i=0}^{N} \bar{H}^{i}}
$$

The hydraulic turbines and governor equations are simplified by modeling all the hydraulic power stations of an area as one aggregated hydraulic turbine and governor. The dynamics of the turbine can in turn be represented by a linearized version of the nonlinear model used in the proxy system, where 
the reference points for the PM system states are used for linearization. This gives the following dynamic equation for the aggregated turbine of area $i$

$$
\begin{aligned}
\Delta \dot{\bar{q}}^{i} & =-\frac{2}{\bar{T}_{w}^{i} \bar{c}_{s s}^{2} / \bar{q}_{s s}}\left(\Delta \bar{q}^{i}-\frac{\bar{q}_{s s}^{i 2}}{\bar{c}_{s s}^{i 2}} \Delta \bar{c}^{i}\right) \\
\Delta \bar{P}_{m}^{i} & =\bar{A}_{t}^{i} \bar{h}_{s s}^{i}\left(3 \Delta \bar{q}^{i}-2 \frac{\bar{q}_{s s}^{i 2}}{\bar{c}_{s s}^{i 2}} \Delta \bar{c}^{i}\right)
\end{aligned}
$$

where $h$ is the pressure head of the penstock, $\bar{T}_{w}$ the water starting time, $A_{t}$ a factor that accounts for the different per-unit bases of the turbine and the governor, and $\Delta \bar{c}$ and $\Delta \bar{q}$ the change in valve opening and water flow rate from $\bar{c}_{s s}$ and $\bar{q}_{s s}$, respectively. In the governor equations, one of the time constants is several times smaller than the others, and it can therefore be neglected. This results in the following governor dynamic equations for area $i$

$$
\begin{aligned}
& \Delta \dot{\bar{\xi}}_{2}^{i}=-\frac{1}{\bar{T}_{r}^{i}} \Delta \bar{\xi}_{2}^{i}+\Delta \bar{c}^{i} \\
& \Delta \dot{\bar{\xi}}_{3}^{i}=\operatorname{sat}_{\dot{c}}^{i}\left(\frac{1}{\bar{T}_{g}^{i}}\left(\Delta \bar{c}_{r}^{i}-\Delta \bar{f}^{i}+\frac{\bar{r}^{i}}{\bar{T}_{r}^{i}} \Delta \bar{\xi}_{2}^{i}-\left(\bar{r}^{i}+\bar{\rho}^{i}\right) \Delta \bar{c}^{i}\right)\right) \\
& \Delta \bar{c}^{i}=\operatorname{sat}_{c}^{i}\left(\Delta \bar{\xi}_{3}^{i}\right)
\end{aligned}
$$

where $\bar{r}$ and $\bar{\rho}$ are the transient and static droop coefficients, $\bar{T}_{r}$ and $\bar{T}_{g}$ time constants, and $\operatorname{sat}_{\dot{c} / c}(\cdot)$ saturations. The manipulated input is still the valve opening set point $\Delta \bar{c}_{r}$.

\section{Disturbance Model}

The balancing of power is managed by market transactions in most systems, and through these markets the predicted power demand and supply are balanced on slots of normally one hour. The markets take into account variations in production from traditional power stations as well as the daily/weekly/annually deterministic load variation patterns. Because

- load variation patterns as well as production from some generators are difficult to predict in detail, and

- changes in production set-point $P_{m, s p}$ takes place on the hour, while changes in power demand happens during the hour (Statnett, 2012) 
there will always be deviations from the hourly market balance. In order to keep the power system safe and stable, it is important that this unpredicted power imbalance is covered by the primary control and the AGC.

By choosing the initial steady state of the PM as the marked balance, the disturbance of the PM $\Delta P_{L}^{i}$ represents the total unpredicted power imbalance of each area, and when dealing with power systems including a certain amount of wind power, such as the Nordic grid, one can for simplicity assume that it is dominated by the fluctuations in produced wind power. If, in addition, it is assumed that the majority of wind power is situated in area $p, \Delta \bar{P}_{L}$ of all the other areas can be neglected, and the model is affected by one single disturbance $\Delta \bar{P}_{L}=\Delta \bar{P}_{L}^{p}$. With the Nordic network in mind, Denmark and South Sweden contribute with about $80 \%$ of the total wind power production (Statnett, 2012). In Ersdal et al. (2015) the disturbance $\Delta \bar{P}_{L}$ was kept constant over the prediction horizon. In this work however, the idea from Ersdal et al. (2014) to make the NMPC more robust against fluctuations in produced wind power by using different disturbance scenarios in the predicted system behavior, is implemented.

Depending on the number of areas $N$, the simplified system is represented by one differential equation

$$
\dot{\bar{x}}=\bar{f}(\overline{\mathbf{x}}, \overline{\mathbf{u}}, \overline{\mathbf{w}})
$$

where

$$
\begin{aligned}
\overline{\mathbf{x}} & =\left[\begin{array}{lllll}
\Delta \bar{f}^{i} & \Delta \bar{q}^{i} & \Delta \bar{\xi}_{2}^{i} & \Delta \bar{\xi}_{3}^{i} & \Delta \bar{P}_{t i e}^{i}
\end{array}\right]^{T} \\
\overline{\mathbf{u}} & =\Delta \bar{c}_{r}^{i} \\
\overline{\mathbf{w}} & =\Delta \bar{P}_{L}^{i}
\end{aligned}
$$

and $i=1, \cdots, N$. In total $5 N$ dynamic state variables, $N$ controllable inputs, and one disturbance. The bar notation represents the simplified system states, inputs and parameters.

\section{Controller}

\subsection{Control Problem}

The main control task of the MPC is to balance the power production in the network against the consumption at all times, and to do so while avoiding bottleneck congestions and staying within other system bounds. When 
large, unexpected disturbances appear, it is a problem in the Nordic network that some of the resources participating in primary control and AGC are prevented from participating due to bottlenecks in the network. For instance, the generators in the west may not be able to participate in covering a disturbance in the east if the east-west transmission lines are already transferring close to maximum.

In addition to this primary control task, the MPC should also keep the costs associated with LFC at a minimum. The cost of primary control is often higher than AGC, and lowering the use of primary control by keeping the frequency closer to $f_{n}$ through use of AGC is desirable. When it comes to use of AGC, it is advantageous to keep the input in the PM $\bar{u}$ as close to its hourly set-point value $\bar{u}_{0}$ as possible. This is because the TSO has to pay more per delivered MWh the more they require the generators to deviate from the initial hourly set point. In Ersdal et al. (2015) it was shown how a NMPC can lower the costs associated with LFC compared to tradition PIcontrol. In this work the aim is not to further improve the reserve usage, but rather expand the NMPC while maintaining the results from Ersdal et al. (2015).

\section{2. $M P C$}

MPC is a framework for advanced control that has seen widespread use, especially within chemical process industries, and it is believed that the optimizing and constraint-handling nature of MPC makes it suitable also for LFC. MPC uses a model of the system to predict how it will behave in the future, and then optimizes the controlled input with regards to an objective function measuring predicted performance. Mathematically, it can be formulated as a continuous time optimal control problem on the form (10a) subject to $(10 \mathrm{~b})$ - (10d) (Biegler, 2010)

$$
\begin{aligned}
\min _{\overline{\mathbf{x}}(\cdot), \overline{\mathbf{u}}(\cdot)} J(\overline{\mathbf{x}}(t), \overline{\mathbf{u}}(t)) & \\
\overline{\mathbf{x}}(0)-\overline{\mathbf{x}}_{0} & =0 \text { Fixed initial state } \\
\dot{\overline{\mathbf{x}}}(t)-f(\overline{\mathbf{x}}(t), \overline{\mathbf{u}}(t), \overline{\hat{\mathbf{w}}}(t)) & =0 \text { System model } \\
g(\overline{\mathbf{x}}(t), \overline{\mathbf{u}}(t)) & \leq 0 \text { Constraints }
\end{aligned}
$$

where $\overline{\mathbf{x}}(t)$ are the system states, $\overline{\mathbf{u}}(t)$ the controlled inputs, $\hat{\overline{\mathbf{w}}}(t)$ the predicted disturbances, and $J(\overline{\mathbf{x}}(t), \overline{\mathbf{u}}(t))$ the control objective function. 


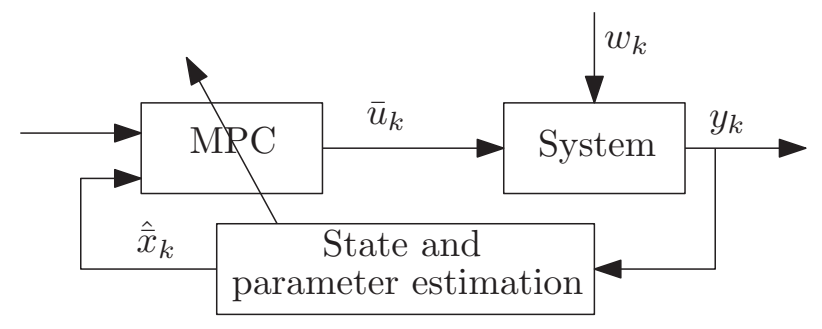

Figure 6: Overview of MPC-loop.

Fig. 6 shows the basics of how an MPC works. The idea is to solve an optimization problem at each time step to find the optimal system input $\overline{\mathbf{u}}(t)$ over a fixed time horizon with respect to the objective function $J(\cdot)$, and then apply the first element of $\overline{\mathbf{u}}(t)$ as input to the system. The loop is closed by the measurements $\mathbf{y}(t)$, and a state estimator is also included as the MPC needs knowledge of the entire state vector $\overline{\mathbf{x}}(t)$.

\section{3. $M P C$ Design for $A G C$}

An MPC will be applied for AGC in this work. It will control the proxy system of Section 2.1, from now referred to as the plant replacement model (PRM), while using the prediction model (PM) of Section 2.2 to predict future system behavior. Hence there is a natural model-plant mismatch between PM and PRM. The control of, and contribution from tertiary control is omitted in this work, as it is manually operated over a longer time scale.

Since the saturations in (7) are nonlinear, a nonlinear MPC (NMPC) must be used, and the optimization problem is therefore non-convex. The continuous time optimization problem (10) in the NMPC is solved with direct methods, that is, it is discretized and transformed into a nonlinear program (NLP) (Biegler, 2010) using collocation and the Casadi framework (Andersson, 2013). Limitations on generation capacity, generation rate of change, as well as on tie-line power transfer are included in the NMPC.

In the PM, there is one input per area, which leads to $N$ optimal inputs from the NMPC: $\Delta \bar{c}_{r}^{i}$, where $i=1, \cdots, N$. These $N$ inputs are then distributed to the hydro generators of each area by individual participation factors $\alpha_{j}^{i}$. As seen from Figure 7

$$
c_{r j}^{i}=c_{r 0 j}^{i}+\alpha_{j}^{i} \Delta \bar{c}_{r}^{i}
$$

where $j=1, \cdots, m_{h}^{i}$ and $m_{h}^{i}$ is the number of hydro generators in area $i$. Bear in mind that $\sum_{i=0}^{N} m_{h}^{i}=m_{h}$. However, each of the valve set points 


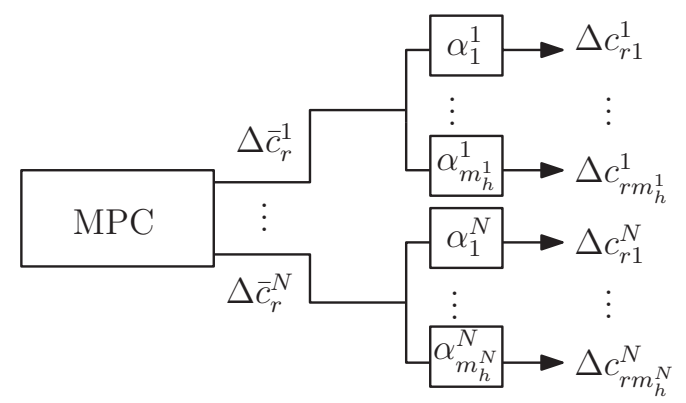

Figure 7: Participation factors $\alpha_{j}^{i}$, where $m_{h}^{i}$ is the number of hydro generators in area $i$.

of the PRM $c_{r}$ has individual constraints, and in Ersdal et al. (2015) the participation factors $\alpha$ were included in the optimization problem as optimization variables. This both allows for individual input constraints and greater flexibility and better use of the NMPC's strength on coordination of multiple inputs, however at the expense of a larger optimization problem. This extension of the PM is also included in this work, and the PM now has $5 N$ dynamic states, $m_{h}$ optimization variables and one disturbance.

\subsection{State and Parameter Estimation}

The NMPC relies on knowledge of the entire PM state vector $\overline{\mathbf{x}}$. It is assumed in this work that the PRM measurements are $\mathbf{y}=\left[|U|, \theta, \Delta f, z_{2}, c, \Delta P_{\text {tie }}\right]$. Based on this, the PM state vector $\overline{\mathbf{x}}$ and disturbance $\overline{\mathbf{w}}=\Delta \bar{P}_{L}$ are estimated using an extended Kalman filter (EKF) (Simon, 2006) which is based on the PM.

\section{Robustified NMPC}

\subsection{Worst-Case Estimate of $\Delta P_{L}$}

For a given wind farm, a worst-case estimate of the variation from the predicted power output can be estimated, and Holttinen (2004) provides such data one second, one minute and one hour into the future $t$. If this information is combined for several wind farms, one can create a total worstcase estimate for the future variation in wind-power production of one area, see Figure 8.

If the wind farms are distributed more evenly in the system, so that the assumption in Section 2.2 no longer applies, the disturbance would enter 


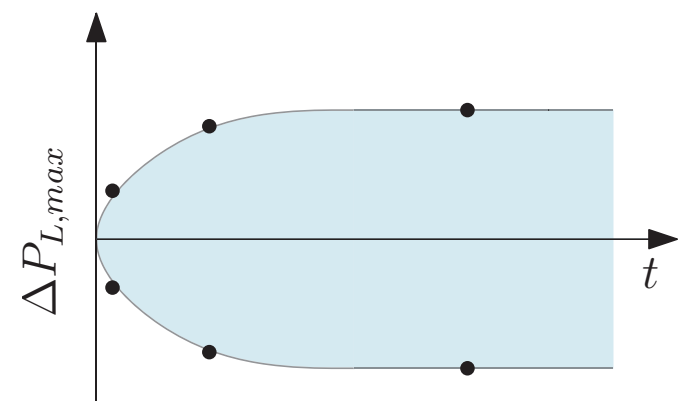

Figure 8: An example of how a worst-case estimate for $\Delta P_{L}$ could look.

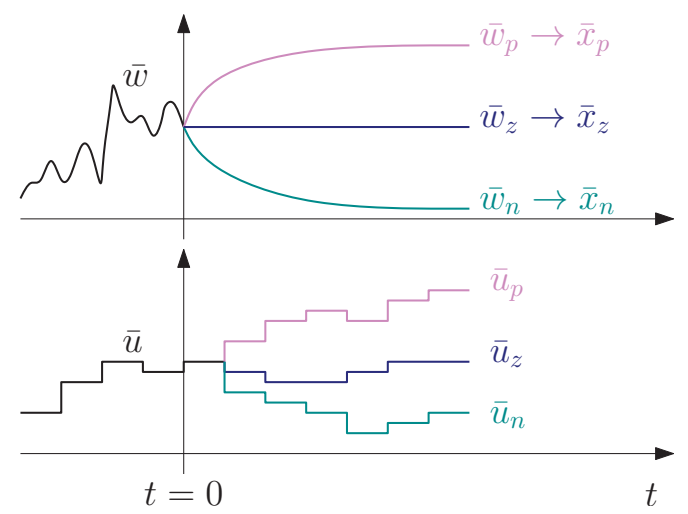

Figure 9: Robustified NMPC. For simplicity, $\bar{u}$ is considered to be scalar.

at several points and a worst case combination of the disturbances, where system dynamics are considered, has to be calculated.

\subsection{Robustified $N M P C$}

In the RNMPC a new state vector is constructed by combining the original state vector $\overline{\mathbf{x}}(9 \mathrm{a})$ and input $\overline{\mathbf{u}}(9 \mathrm{~b})$ exposed to three different future disturbances: The zero, negative, and positive worst-case estimate for $\Delta P_{L}$ added to the current estimated disturbance $\overline{\mathbf{w}}(9 \mathrm{c})$, see Figure 9 . The system equation for the combined system used in (10c) is

$$
\dot{\mathbf{x}}=\left[\begin{array}{c}
\dot{\mathbf{x}}_{z} \\
\dot{\overline{\mathbf{x}}}_{p} \\
\dot{\overline{\mathbf{x}}}_{n}
\end{array}\right]=\left[\begin{array}{c}
f\left(\overline{\mathbf{x}}_{z}, \overline{\mathbf{u}}_{z}, \overline{\mathbf{w}}_{z}\right) \\
f\left(\overline{\mathbf{x}}_{p}, \overline{\mathbf{u}}_{p}, \overline{\mathbf{w}}_{p}\right) \\
f\left(\overline{\mathbf{x}}_{n}, \overline{\mathbf{u}}_{n}, \overline{\mathbf{w}}_{n}\right)
\end{array}\right]=f(\mathbf{x}, \mathbf{u}, \mathbf{w})
$$


where $\mathbf{u}=\left[\begin{array}{lll}\overline{\mathbf{u}}_{z} & \overline{\mathbf{u}}_{p} & \overline{\mathbf{u}}_{n}\end{array}\right]^{T}$ and $\mathbf{w}=\left[\begin{array}{lll}\overline{\mathbf{w}}_{z} & \overline{\mathbf{w}}_{p} & \overline{\mathbf{w}}_{n}\end{array}\right]^{T}$. At each optimization it is required that for the first element of $\mathbf{u}(t), \overline{\mathbf{u}}_{z}, \overline{\mathbf{u}}_{n}$ and $\overline{\mathbf{u}}_{p}$ must be equal. After this, they are free to vary in manners optimal for their designated system states, see Figure 9. In this way every input guarantees that the optimal input trajectory for all three scenarios, fulfilling all system constraints, are still feasible.

\subsection{Stability of the RNMPC}

A common approach for achieving robust NMPC is the concept of minmax NMPC (Rawlings and Mayne, 2009), which often results in large and complex optimization problems. The RNMPC presented in the previous section is a simplified, nonlinear version of the min-max feedback MPC presented in Scokaert and Mayne (1998). It is well known that min-max approaches are computationally intractable, especially in the nonlinear case. This motivates us to only consider predefined approximate worst case scenarios instead of letting the optimizer find the real worst case disturbance, which is an approach that is not very different from what is done in scenariobased NMPC (Huang et al., 2009; Goodwin and Medioli, 2013). A drawback with doing this is however that the recursive feasibility property is lost. This is important in proving closed-loop stability of MPC schemes (Mayne et al., 2000), and stability of the RNMPC can therefore not be guaranteed. Stability is rather approached by choosing long horizons and tuning weights to achieve convergent behavior in simulations. "Stabilizing ingredients" such as terminal costs could have been added, but this is not included in this study.

To handle possible instability/infeasible optimization problems, a supervising agent must be devised that interferes when problems are detected, as a fallback mechanism. This could involve automatic fail-to-safe transitions, possible restart of the MPC, or switching to manual control. It can be argued that such a mechanism will have to be installed in any case, since it is very hard (and conservative) to include all thinkable disturbance scenarios in the prediction model. The role of the robustified MPC is then to handle the disturbances most likely to happen.

\section{Case Study}

The PRM (1) has a total of 100 dynamic states, 72 algebraic states, 15 controllable inputs, and 21 disturbances. This system is divided into two areas in the PM (8), hence $N=2$. One area covers South Sweden and 
Eastern Denmark (area A), and the other covers Norway, North Sweden and Finland (area B), and according to the assumptions made in Section 2.2, $\Delta \bar{P}_{L}^{B}=0$ and $\Delta \bar{P}_{L}=\Delta \bar{P}_{L}^{A}$. There is also a tie line between the two areas which represents the total power flow between them. This tie line has a maximum power-transfer capacity of $2000 \mathrm{MW}$. With $N=2$, the PM has 10 dynamic sates, 15 controllable inputs, and one disturbance, which is a significant reduction from the PRM. The AGC control signal is dispatched every $10 \mathrm{~s}$ for both the PRM and the PM.

The performance of the RNMPC is compared to the NNMPC, which is based on $\dot{\bar{x}}_{z}=\bar{f}\left(\bar{x}_{z}, \bar{u}_{z}, \bar{w}_{z}\right)$. In order to compare the two controllers, a control performance measure (CPM) is applied. It is inspired by the CPS1 and CPS2 performance criteria used by the North American electric reliability corporation (NERC) (Gross and Lee, 2001), and it basically measures the average frequency deviation: first $\Delta f$ is averaged over windows of $30 \mathrm{~s}$ to filter out fast fluctuations, then the CPM is found by again averaging $\Delta f$ over all these windows.

\subsection{Tuning the $N M P C$}

The main tuning variables of the NMPC are the prediction horizon $T$, and the objective function $J(\cdot)$. The objective function is set to

$$
J(\overline{\mathbf{x}}, \overline{\mathbf{u}})=\int_{t=0}^{T} \overline{\mathbf{x}}^{T} \mathbf{Q} \overline{\mathbf{x}}+\left(\overline{\mathbf{u}}-\overline{\mathbf{u}}_{0}\right)^{T} \mathbf{R}\left(\overline{\mathbf{u}}-\overline{\mathbf{u}}_{0}\right) d t
$$

where $\mathbf{Q}=\operatorname{diag}\left(\overline{\mathbf{Q}}_{z}, \overline{\mathbf{Q}}_{p}, \overline{\mathbf{Q}}_{n}\right)$ and $\mathbf{R}=\operatorname{diag}\left(\overline{\mathbf{R}}_{z}, \overline{\mathbf{R}}_{p}, \overline{\mathbf{R}}_{n}\right)$ for the RNMPC, and $\mathbf{Q}=\overline{\mathbf{Q}}$ and $\mathbf{R}=\overline{\mathbf{R}}$ for the NNMPC. $\mathbf{Q}$ is real, symmetric and positive semidefinite, while $\mathbf{R}$ is real, symmetric and positive definite. The non-zero elements of $\overline{\mathbf{Q}}$ are chosen so that the deviation in overall system frequency (5) is punished: $\bar{q}_{11}=\beta \frac{\left(H^{1}\right)^{2}}{\left(H^{1}+H^{2}\right)^{2}}, \bar{q}_{66}=\beta \frac{\left(H^{2}\right)^{2}}{\left(H^{1}+H^{2}\right)^{2}}, \bar{q}_{16}=\bar{q}_{61}=\beta \frac{H^{1} H^{2}}{\left(H^{1}+H^{2}\right)^{2}}$, where $\beta=10^{5}$. The use of primary control is actuated by $\Delta f$, so minimizing $\Delta f$ will also minimize the use of primary reserves. The matrix $\mathbf{R}$ is set to $\mathbf{R}=\operatorname{diag}\left(\eta \mathbf{m}_{\text {base }}\right)$, where $\mathbf{m}_{\text {base }}$ is a vector containing the hydro generators' base rating, and $\eta=0.1$. With $\mathbf{m}_{\text {base }}$ included in $\mathbf{R}$, the actual produced power from the hydro generators are included in the objective function, and not only the per-unit based $\overline{\mathbf{u}}$. In order to place more emhasis on deviations in $\overline{\mathbf{x}}_{z}, \overline{\mathbf{Q}}_{z}$ is set equal to $\overline{\mathbf{Q}}$, while $\overline{\mathbf{Q}}_{p}=\overline{\mathbf{Q}}_{n}=0.1 \overline{\mathbf{Q}}$. The three systems are all punished equally when it comes to deviations in input: $\overline{\mathbf{R}}_{z}=\overline{\mathbf{R}}_{p}=\overline{\mathbf{R}}_{n}=\overline{\mathbf{R}}$. 


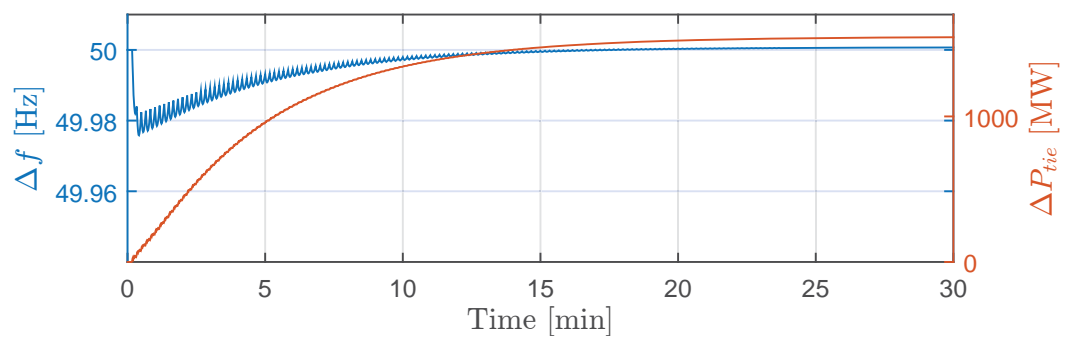

(a) Nominal NMPC

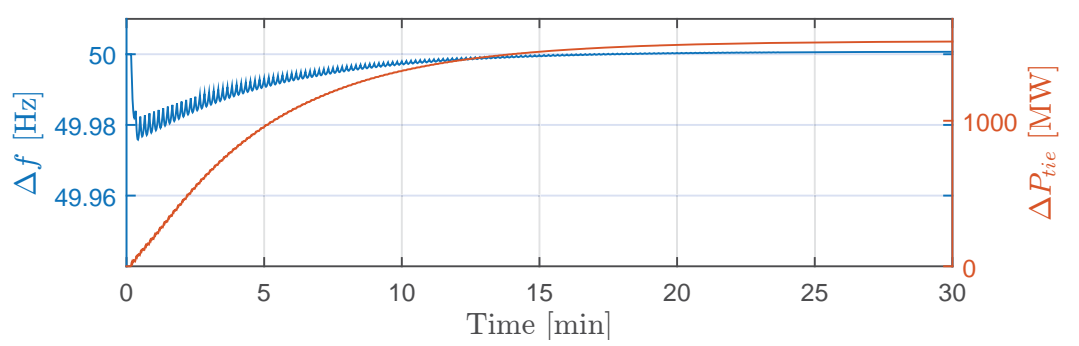

(b) Robustified NMPC

Figure 10: Case A: $\Delta f$ and $\Delta P_{t i e}$ for NNMPC and RNMPC.

The control horizon $T$ is 3 minutes, a decision based on a compromise between system time constants and complexity, and the time step of the NMPCs is $10 \mathrm{~s}$ in order to match the control signal dispatching in the system.

\subsection{Tests on the SINTEF proxy system}

The NMPC has been tested on the SINTEF Nordic system model described earlier. As discussed, it is normal practice in power system research to use high fidelity validated models to test operational and research concepts since the transmission system itself cannot be used for experimentation.

In Case $\mathrm{A}$, the disturbance (entering in area $\mathrm{A}$ ) is small enough so that $\Delta P_{\text {tie }}$ does not reach its limit. Figure 10 shows the resulting $\Delta f$ and $\Delta P_{\text {tie }}$, and it is clear that in this case the response is very similar for the NNMPC and the RNMPC. This is confirmed by the CPM in Table 1. It is also seen from Figure 10 that there are oscillations in the system frequency. These oscillations are mainly caused by the the fast, electromechanical dynamics of the system, which the MPC is not able to account for due to turbine time constants and MPC sampling time.

In Case $\mathrm{B}$ the initial state of the system has $\Delta P_{L} \neq 0$ (and thus $\Delta P_{\text {tie }} \neq$ 0 ), and after approximately 4 minutes, the disturbance (still entering in area 
Table 1: CPM and reserves usage (given in MWh).

\begin{tabular}{|c|c|c|c|c|}
\hline & & CPM & Primary Control & $\mathrm{AGC}$ \\
\hline \multirow{3}{*}{ Case A } & NNMPC & $3.73 \cdot 10^{-5}$ & 26.0 & 768.7 \\
\hline & RNMPC & $3.75 \cdot 10^{-5}$ & 25.9 & 769.2 \\
\hline & Difference & $+0.5 \%$ & $-0.4 \%$ & $\approx \pm 0 \%$ \\
\hline \multirow{3}{*}{ Case B } & NNMPC & $1.28 \cdot 10^{-3}$ & 88.2 & 2525 \\
\hline & RNMPC & $1.27 \cdot 10^{-3}$ & 89.4 & 2518 \\
\hline & Difference & $-0.8 \%$ & $+1 \%$ & $-0.3 \%$ \\
\hline \multirow{3}{*}{ Case C } & NNMPC & $3.5 \cdot 10^{-3}$ & 2298 & 746 \\
\hline & RNMPC & $3.9 \cdot 10^{-3}$ & 2463 & 777 \\
\hline & Difference & $+11 \%$ & $+7 \%$ & $+4 \%$ \\
\hline
\end{tabular}

A) follows the positive worst-case scenario of Figure 8. The test results can be seen in Figure 11 - 13. Figure 11(b) shows that in the initial steady state, the limit of $\Delta P_{t i e}$ is reached by the NNMPC, while the RNMPC stabilizes the tie-line power transfer at a lower level, ensuring that there still is some transfer capacity left. This is very beneficial when the worst-case disturbance sets in, and Figure 11(b) clearly shows that the RNMPC manages to keep within the limit of 2000 MW while the NNMPC does not. Figure 12 shows how $\Delta P_{m}$ is allocated between the two areas with the NNMPC and the RNMPC, and in Figure 13 the total input $\sum u=\sum c_{r}$ of each area can be seen.

It is clear from both Figure 11 and 12 that the NNMPC experiences larger system oscillations than the RNMPC. This is because the NNMPC is at the tie-line constraint, causing the inputs to constantly having to adjust in an attempt to fulfill the system constraints, see Figure 13(a). These input oscillations are not seen with the RNMPC (Figure 13(b)), and they cause oscillations in generated power as well as amplifying the oscillations in system frequency. They are not beneficial for the hydro generators as they will cause wear and tear on their valves and other mechanical parts.

The last case, Case C, is a Monte Carlo simulation where disturbances based on random numbers, generated using the method of Cecílio et al. (2013) are imposed on the system. These disturbances are much more fluctuating 


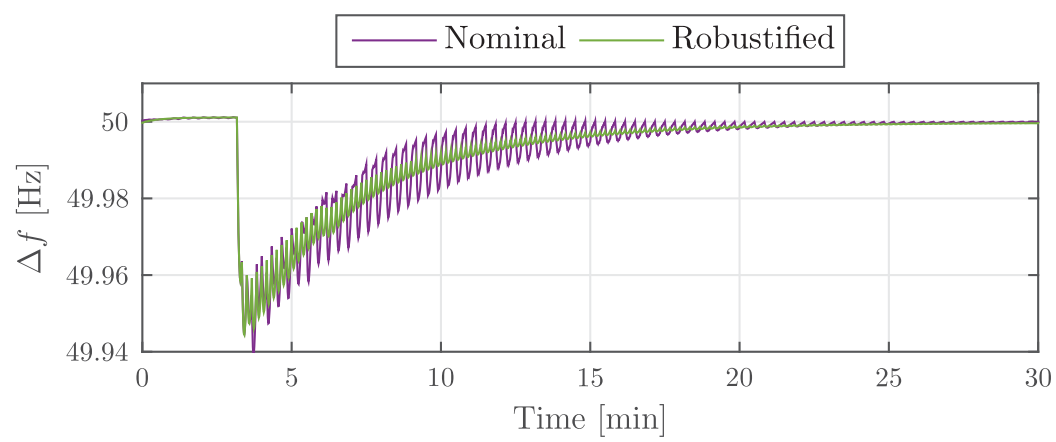

(a) $\Delta f$

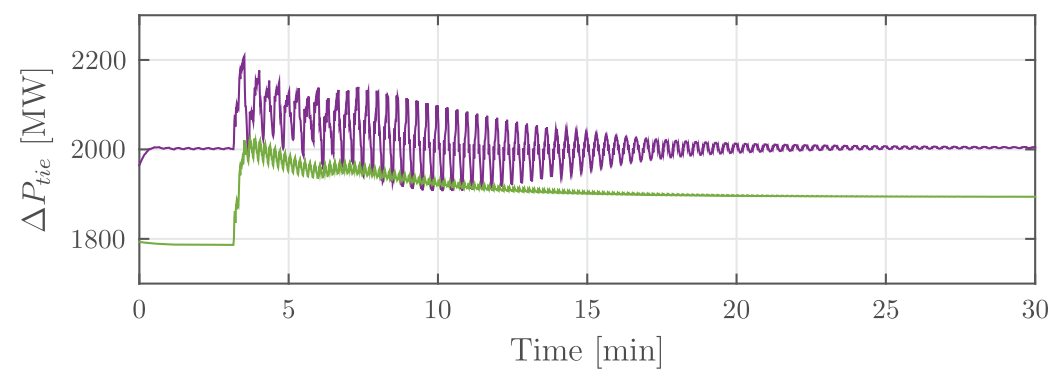

(b) $\Delta P_{t i e}$

Figure 11: Case B: $\Delta f$ and $\Delta P_{t i e}$ for NNMPC and RNMPC. 


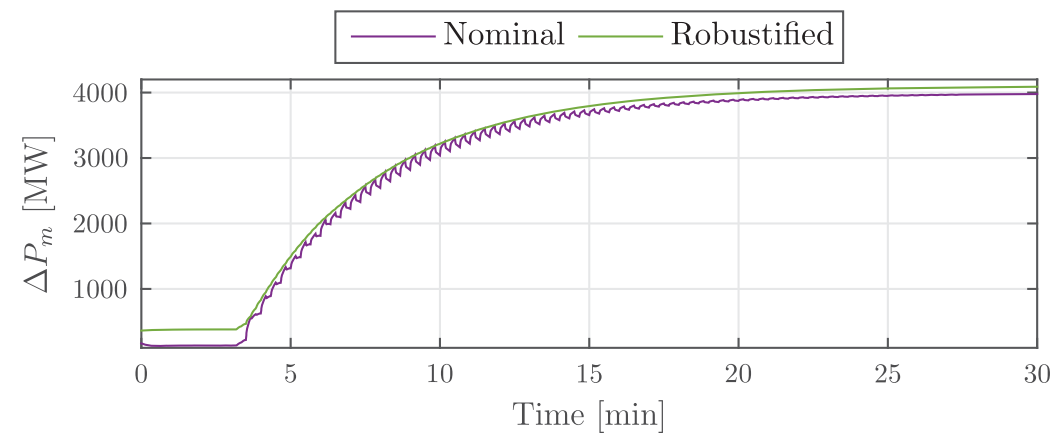

(a) $\Delta P_{m}$ in area $\mathrm{A}$.

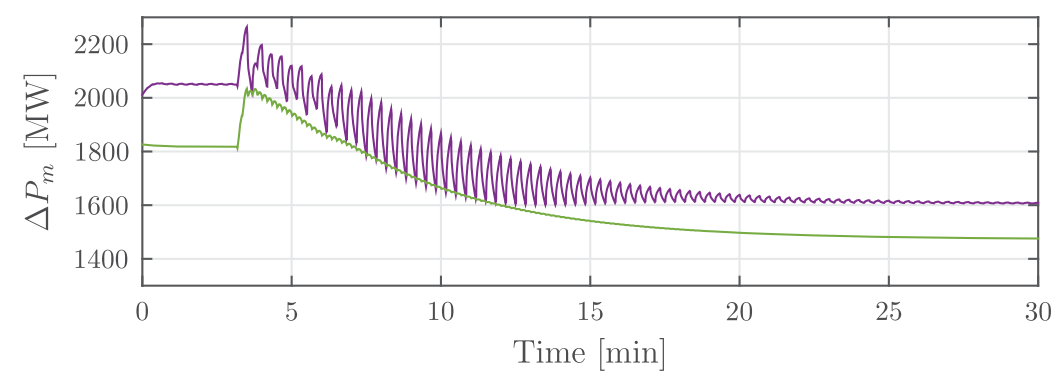

(b) $\Delta P_{m}$ in area $\mathrm{B}$.

Figure 12: Case B: total use of $\Delta P_{m}$ in area $\mathrm{A}$ and area $\mathrm{B}$ with nominal and robustified controller. 


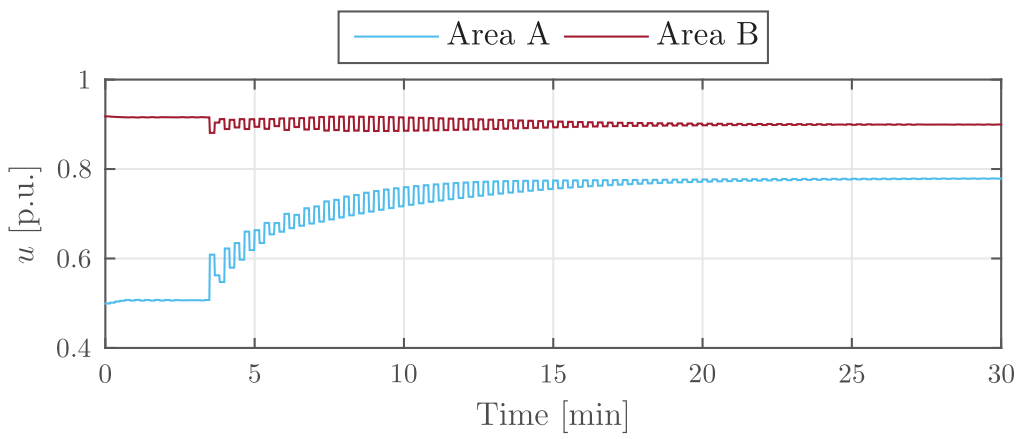

(a) $\sum u=\sum c_{r}$ in area $\mathrm{A}$ and $\mathrm{B}$ with NNMPC.

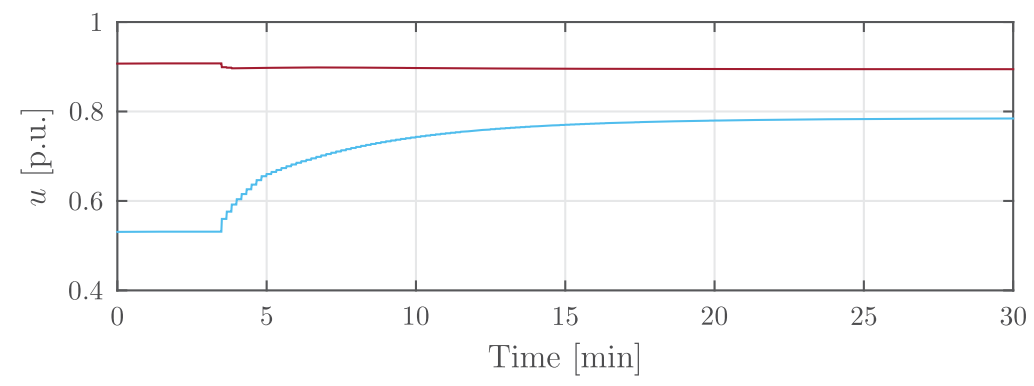

(b) $\sum u=\sum c_{r}$ in area A and B with RNMPC.

Figure 13: Case B: total input in area A and area B with nominal and robustified controller. 
and smaller in size compared to the previous cases, so that they resemble normal disturbance patterns. The Monte Carlo simulation is included to show the average performance of the RNMPC against the NNMPC, and the average results from 100 simulations can be seen in Table 1. It shows that the NNMPC in general performs slightly better than the RNMPC, both with regards to frequency control and reserve usage. This is however not very surprising, as robust control methods tends to be more conservative, which often leads to poorer control performance.

\section{Discussion}

In Ersdal et al. (2015) it was shown through both descriptive examples and thorough simulation, that applying MPC for AGC can be beneficial with regards to control performance and reserves usage. In this work, the emphasis has been placed on making the NMPC presented in Ersdal et al. (2015) more robust against fluctuating wind power production.

In case $\mathrm{A}$ it was demonstrated that when no system constraints are met, the NNMPC and RNMPC results in almost identical system behavior and reserve usage. On average however, the Monte Carlo simulation showed that the NNMPC does perform better than the RNMPC while using less resources. This is however something that must be expected when trying to robustify the NMPC. Another result from the Monte Carlo simulation is data on how often the RNMPC results in infeasible optimization problems. For 100 successful simulations, there where 12 that ended with infeasibility (hence 11\%). With the NNMPC, the tie-line constraints were broken in these cases, and the only reason why infeasibility was not encountered is that the NNMPC has slack variables to handle this. Adding slack variables to system constraints is a common way of avoiding infeasibility in optimization problems (Maciejowski, 2002), as they allow the solution to break certain constraints if absolutely necessary. Slack variables were not inlcuded in the RNMPC because they would deteriorate the aim of keeping clearances to system constraints.

Even though the RNMPC showed to be more conservative when more realistic, fluctuating disturances were imposed on the system, it is still less conservative than the RNMPC in Ersdal et al. (2014). The main reason for this difference is that in Ersdal et al. (2014) there was only one input per area, whereas here there are several. Several inputs per area makes it easier for the RNMPC to fulfill the system constraints, also in case of worst-case disturbance scenarios, and it does not need to be as conservative. 
In case $\mathrm{B}$ the difference between the NNMPC and RNMPC is displayed. When the disturbance is at a level so that $\Delta P_{\text {tie }}$ approaches its limit, the NNMPC will stabilize $\Delta P_{\text {tie }}$ at the limit, while the RNMPC will keep some clearance to it in case of a new worst-case disturbance. And as seen from Figure 11(b), the RNMPC manages to keep within the tie-line limit when the worst-case disturbance sets in, while the NNMPC does not. In Figure 12 it is seen how the NNMPC and RNMPC allocates $\Delta P_{m}$ in area $\mathrm{A}$ and area $\mathrm{B}$ differently. It shows that the two controllers gives similar results, only that the RNMPC in general allocates more of $\Delta P_{m}$ to area $\mathrm{A}$ where the disturbance enters, resulting in a lower $\Delta P_{t i e}$. Both Figure 11(a) and Table 1 shows that the control performance of the NNMPC and RNMPC in case $\mathrm{B}$ are very similar, and there is some increase in the use of primary control while the use of AGC is lowered. However, these are very small numbers, and for all practical purposes the two controllers performs equally with regards to frequency restoration and reserves usage.

\section{Conclusion}

The negative trend seen in the quality of LFC in the Nordic network over the last decades indicates that something must be done to better cope with the challenges of intermittent energy resources and network bottlenecks. In Ersdal et al. (2015) it was shown how applying NMPC for AGC can improve the LFC by better coordination of AGC and primary control, as well as including power-transfer limits as constraints in the MPC. In this work, this has been extended to include the robustified NMPC (RNMPC) from Ersdal et al. (2014), and simulations show that also with a more realistic simulation setup (including model-plant mismatch and state estimation), the RNMPC manages to keep transfer capacity available for severe situations of unpredicted fluctuations in produced wind power from the area of South Sweden and East Denmark. The behavior of the RNMPC was compared to that of the nominal NMPC (NNMPC) from Ersdal et al. (2015), and Monte Carlo simulations show that the NNMPC does perform better than the RNMPC on average. However, the NNMPC is not able to avoid bottlenecks in the same way as the RNMPC, and there will almost always be a trade off between system performance and robustness of a controller. The difference in performance and reserve usage is however not deterrent, and this work that the scheme presneted here could be a realistic way of dealing with bottlenecks in the Nordic network, ensuring available transfer capacity so that all available 
resources are able to participate in the $\mathrm{LFC}$.

\section{Acknowledgements}

The financial support from the Research Council of Norway project 207690 Optimal Power Network Design and Operation, and the Marie Curie FP7IAPP project Using real- time measurements for monitoring and management of power transmission dynamics for the Smart Grid - REAL-SMART Contract No: PIAP-GA-2009- 251304, are gratefully acknowledged.

\section{References}

Andersson, J., 2013. A General-Purpose Software Framework for Dynamic Optimization. PhD thesis, Arenberg Doctoral School, KU Leuven.

Bevrani, H., 2009. Robust Power System Frequency Control. Springer.

Bevrani, H., Hiyama, T., Bevrani, H., 2011. Robust PID based power system stabilizer: Design and real-time implementation. International Journal of Electrical Power \& Energy Systems 33, 179-188.

Biegler, L. T., 2010. Nonlinear Programming: Concepts, Algorithms, and Applications to Chemical Processes. SIAM.

Carneiro, J., Ferrarini, L., Nov 2010. Preventing thermal overloads in transmission circuits via model predictive control. Control Systems Technology, IEEE Transactions on 18 (6), 1406-1412.

Çam, E., Kocaarslan, I., Jan 2005. Load frequency control in two area power system using fuzzy logic controller. Energy Conversion Management 46, 233-243.

Cecílio, I. M., Ersdal, A. M., Fabozzi, D., Thornhill, N. F., 2013. An opensource educational toolbox for power system frequency control tuning and optimization. In: Innovative Smart Grid Technologies Europe (ISGT EUROPE), 2013 4th IEEE/PES. IEEE.

Chang-Chien, L.-R., Lin, W.-T., Yin, Y.-C., May 2011. Enhancing frequency response control by dfigs in the high wind penetrated power systems. Power Systems, IEEE Transactions on 26 (2), 710-718. 
Ersdal, A., Imsland, L., Uhlen, K., 2015. Model predictive load-frequency control. Power Systems, IEEE Transactions on (to appear).

Ersdal, A. M., Fabozzi, D., Imsland, L., Thornhill, N. F., 2014. Model predictive control for power system frequency control taking into account imbalance uncertainty. In: IFAC World Congress. Vol. 19. pp. 981-986.

Fabozzi, D., Thornhill, N. F., Pal, B. C., 2013. Frequency restoration reserve control scheme with participation of industrial loads. In: Proc. 2013 IEEE Grenoble PowerTech conf.

Goodwin, G. C., Medioli, A. M., 2013. Scenario-based, closed-loop model predictive control with application to emergency vehicle scheduling. International Journal of Control 86 (8), 1338-1348.

Gross, G., Lee, J. W., 2001. Analysis of load frequency control performance assessment criteria. Power Systems, IEEE Transactions on 16, 520-525.

Halvgaard, R., Poulsen, N. K., Madsen, H., Jorgensen, J. B., 2012. Economic model predictive control for building climate control in a smart grid. In: Innovative Smart Grid Technologies (ISGT), 2012 IEEE PES. IEEE, pp. $1-6$.

Holttinen, H., 2004. The impact of large scale wind power production on the nordic electricity system. Ph.D. thesis, Helsinki University of Technology.

Huang, R., Patwardhan, S. C., Biegler, L. T., 2009. Multi-scenario-based robust nonlinear model predictive control with first principle models. Computer Aided Chemical Engineering 27, 1293-1298.

Khodabakhshian, A., Edrisi, M., 2008. A new robust PID load frequency controller. Control Engineering Practice 16, 1069-1080.

Langson, W., Chryssochoos, I., Rakovi, S., Mayne, D., 2004. Robust model predictive control using tubes. Automatica 40 (1), 125 - 133.

Löfberg, J., 2003. Minimax approaches to robust model predictive control. Ph.D. thesis, Linköping University.

Machowski, J., Bialek, J. W., Bumby, J. R., 2008. Power System Dynamics. Stability and Control. Wiley. 
Maciejowski, J. M., 2002. Predictive Control with Constraints. Pearson Education.

Marinovici, L., Lian, J., Kalsi, K., Du, P., Elizondo, M., Aug 2013. Distributed hierarchical control architecture for transient dynamics improvement in power systems. Power Systems, IEEE Transactions on 28 (3), 3065-3074.

Mayne, D., Rawlings, J., Rao, C., Scokaert, P., 2000. Constrained model predictive control: Stability and optimality. Automatica 36 (6), $789-814$.

McNamara, P., Negenborn, R. R., De Schutter, B., Lightbody, G., 2013. Optimal coordination of a multiple HVDC link system using centralized and distributed control. Control Systems Technology, IEEE Transactions on $21(2), 302-314$.

Mohamed, T., Bevrani, H., Hassan, A., Hiyama, T., 2011. Decentralized model predictive based load frequency control in an interconnected power system. Energy Conversion and Management 52 (2), 1208 - 1214.

Mohamed, T. H., Morel, J., Bevrani, H., Hiyama, T., dec 2012. Model predictive based load frequency control design concerning wind turbines. International Journal of Electrical Power \& Energy Systems 43 (1).

Nordel, accessed March 2013 2008. Nordel Annual Statistics 1997-2008.

Norheim, I., Lindgren, E., Uski, S., Srensen, P., Jauch, C., 2005. WILMAR WP5 - Deliverable D5.1. System Stability Analysis. Tech. rep., SITNEF Energy Research.

Otomega, B., Marinakis, A., Glavic, M., Van Cutsem, T., 2007. Emergency alleviation of thermal overloads using model predictive control. In: Proc. 2007 IEEE PES PowerTech Conference.

Rawlings, J. B., Mayne, D. Q., 2009. Model Predictive Control: Theory and Design. Nob Hill Publishing.

Saxena, S., Hote, Y., Aug 2013. Load frequency control in power systems via internal model control scheme and model-order reduction. Power Systems, IEEE Transactions on 28 (3), 2749-2757. 
Scokaert, P., Mayne, D., Aug. 1998. Min-max feedback model predictive control for constrained linear systems. IEEE Transactions on Automatic Control 43 (8), 1136-1142.

Shiroei, M., Toulabi, M. R., Ranjbar, A. M., 2013. Robust multivariable predictive based load frequency control considering generation rate constraint. International Journal of Electrical Power \& Energy Systems 46, 405-413.

Short, J., Infield, D., Freris, L., Aug 2007. Stabilization of grid frequency through dynamic demand control. Power Systems, IEEE Transactions on $22(3), 1284-1293$.

Simon, D., 2006. Optimal State Estimation. Wiley.

Singh, V. P., Mohanty, S. R., Kishor, N., Ray, P. K., March 2013. Robust H-infinity load frequency control in hybrid distributed generation systems. International Journal of Electric Power \& Energy Systems 46, 294-305.

Statnett, 2012. Systemdrifts- og markedsutviklingsplan 2012. Tech. rep., available at issuu.com/statnett/docs/statnett_smup.

Suvire, G., Molina, M., Mercado, P., Dec 2012. Improving the integration of wind power generation into ac microgrids using flywheel energy storage. Smart Grid, IEEE Transactions on 3 (4), 1945-1954.

Tan, W., Feb 2010. Unified tuning of pid load frequency controller for power systems via imc. Power Systems, IEEE Transactions on 25 (1), 341-350.

Venkat, A., Hiskens, I., Rawlings, J., Wright, S., Nov 2008. Distributed mpc strategies with application to power system automatic generation control. Control Systems Technology, IEEE Transactions on 16 (6), 1192-1206.

Vrdoljak, K., Perić, N., Petrović, I., 2010. Sliding mode based load-frequency control in power systems. Electric Power Systems Research 80 (5), 514-527.

Whitley, D., Gjerde, O., 2011. LFC/AGC-Nordic and European perspective. In: Exchange of balancing services between the Nordic and the Central European synchronous systems. Available at http://www. sintef.no/globalassets/project/balance-management/gardermoen/ 8---gjerde-statnett---lfc-and-agc---nordic-perspective.pdf . 
Yousef, H., AL-Kharusi, K., Albadi, M., Hosseinzadeh, N., July 2014. Load frequency control of a multi-area power system: An adaptive fuzzy logic approach. Power Systems, IEEE Transactions on 29 (4), 1822-1830. 